\title{
Understanding Mobile Money Grievances from Tweets
}

\author{
Kushal Shah \\ University of Washington \\ kushals@uw.edu
}

\author{
Shrirang Mare \\ University of Washington \\ shri@cs.washington.edu
}

\author{
Richard Anderson \\ University of Washington \\ anderson@cs.washington.edu
}

\begin{abstract}
Access to financial services through a mobile phone, known as Mobile Financial Services (MFS), creates an opportunity to expand the reach of financial services to the 1.7 billion unbanked adults worldwide. Nevertheless, MFS adoption has been inconsistent, which motivates a need to identify the challenges that MFS users confront in different countries. In this work, we explore the Twitter as a potential data source to understand such challenges. More broadly, we assess whether (and how) publicly available Twitter data can augment the findings of expensive, large-scale research studies on MFS barriers. Our Qualitative Content Analysis of 9,000 mobile money grievance tweets that were extracted from 54 MFS customer care twitter feeds across six countries reveals service and access issues, incorrect transactions, and fraud as three main challenges MFS users report on Twitter. We discuss the nuances around these challenges and the substantial differences between the common issues reported in different countries. Ultimately, we conclude that Twitter data can elucidate the challenges of MFS adoption and also that it can augment the results of other types of MFS studies.
\end{abstract}

\section{CCS CONCEPTS}

- Human-centered computing $\rightarrow$ Human computer interaction (HCI); Social media; • Applied computing $\rightarrow$ Sociology;

\section{KEYWORDS}

Mobile Financial Services; ICTD; Twitter; Financial Inclusion

\section{ACM Reference Format:}

Kushal Shah, Shrirang Mare, and Richard Anderson. 2019. Understanding Mobile Money Grievances from Tweets. In Tenth International Conference on Information and Communication Technologies and Development (ICTD '19), fanuary 4-7, 2019, Ahmedabad, India. https://doi.org/10.1145/3287098. 3287123

\section{INTRODUCTION}

Financial inclusion, in which people have access to savings, credit, remittance products, and financial institutions, is recognized as a component of lifting people out of poverty worldwide [1, 10, 34, 39]. Mobile Financial Services (MFS), where access to financial products is through a mobile phone, creates the opportunity for expanding the reach of financial institutions and creating new services which can serve more people [21,37]. Kenya stands out as the notable success, where mobile money, led by M-Pesa, is used by $73 \%$ of

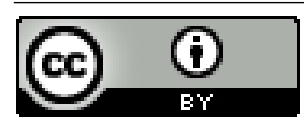

This work is licensed under a Creative Commons Attribution International 4.0 License.

ICTD '19, fanuary 4-7, 2019, Ahmedabad, India

(c) 2019 Copyright held by the owner/author(s).

ACM ISBN 978-1-4503-6122-4/19/01.

https://doi.org/10.1145/3287098.3287123 the adult population. Thus, there is great interest by the development community in understanding the barriers to adoption of these services, both to increase adoption and to support financial inclusion.

Multiple studies seek to understand the barriers for adoption and use of MFS. Generally, these studies involve in-depth interviews with different stakeholders of financial services [19, 38], randomized controlled trials (RCTs) [4], large scale nationally representative surveys $[14,17]$, or longitudinal surveys [14, 21]. While all these types of studies may yield important insights about MFS, the methods are expensive and time-consuming. We explore an opportunity to augment the results of these studies by analyzing Twitter data. Twitter has become a common channel through which customers complain about a service and seek customer care support [2, 12, 20,30]. Many mobile money service providers use customer care Twitter handles where users can report their complaints, ask questions, and seek help regarding the service. Thus, Twitter is potentially a rich dataset about issues reported by mobile money users, and in this paper, we investigate the use of this data for understanding the challenges faced by consumers in adopting MFS in different countries.

The goal of this study is to analyze Twitter data and highlight problems that MFS users in Ghana, India, Kenya, Pakistan, South Africa and Uganda tweet about, and how the corresponding customer service dialogue takes place. We randomly sample 1500 tweets from from all the six countries and then perform qualitative analysis to label the tweets. The tweets were labeled in multiple iterations, the first to identify whether a given tweet was related to MFS and the second to mark the MFS problem. We then condensed our labels into several higher level categories such as service error, transaction reversal, access error, fraud, etc.

We complement this qualitative analysis with other studies undertaken to understand MFS barriers. Our findings indicate that Twitter is a cost-effective and, surprisingly, rich data source to understand problems of MFS users. We find that service issues, where users report transaction delays, and access issues, where they are unable to login, are the most common problems. We then discuss the strengths and weaknesses of our analysis, and provide recommendations to make the findings more robust using cross-cultural analysis and quantitative methods. Finally, we present ways to address the challenges that our analysis highlights.

\section{RELATED WORK}

\subsection{MFS for Financial Inclusion}

Research on MFS ranges from studies that explore financial systems and money to those that directly evaluate the technologies that implement mobile money. Kumar [22] explored that rapidity of transactions, flexibility of bargaining, and complexity of change making that have ramifications for mobile systems. Pal [31] looked at shop-keeper payments in India. O'Neill [29] argues that the 
means of payments is a component of a larger social process. Blumenstock [4] examined that government payments did not go to the beneficiaries. Interview and survey based studies have drawn attention to a wide range of barriers to MFS adoption. Yu [40] identified high transaction costs, technological limitations, and limited need as issues. Ibtasam [19] considers gender and societal barriers as fundamental obstacles. Ghosh [16] draws attention to consumer lack of understanding of financial concepts. Medhi [27] identified agent proximity, transaction costs, and perceived reliability in a multi-country study. Technical work in computing has sought to address specific MFS challenges. Medhi [26] studied how to make mobile money systems more accessible to low-literate. Ibtasam [18] explored the usability and learnability of smartphone mobile wallet applications. Vulnerability of mobile money through "thin-sim" attacks is explored by Phipps [32]. The problem of security of mobile apps is evaluated by Reaves [33], and later by Castle [5].

\subsection{Grievance Redressal}

Mobile technologies have created opportunities for reporting complaints across a wide range of domains. CGNET Swara [24, 28] was developing a mechanism for remote, disconnected users to participate. Marathe [25] studied the scaling of complaint redress. Chakrabory $[6,7]$ has studied the impact of complaints and redress in jobs programs. Gaut [15] looks at automatic methods of complaint classification for appropriate routing. A common theme in works studying complaints is the interplay between supporting technology, human responses, and organizational incentives for resolution.

\subsection{Twitter studies}

Twitter has inspired a breadth of academic research on areas ranging from understanding misinformation [3] to extracting sentiments using automated classification systems [41]. Dunphy [11] sampled 698 tweets with the hashtag "\#password" and then performed Qualitative Content Analysis to identify themes and then label tweets into higher-level categories. Kwizera [23] developed an automated chatbot system for the Kenyan Customer Service Market. Fichet [13] examined how back and forth conversations on Twitter aided in crisis relief.

\section{MFS CHALLENGES}

One of the most significant efforts to understand MSF challenges has been the Financial Inclusion Insights (FII) Program by Intermedia [14]. This includes large scale surveys which have been done annually across Pakistan, India, Tanzania, Uganda, Kenya, Nigeria, Indonesia and Bangladesh to understand financial readiness. The survey asks about mobile money usage and the problems that users face when using mobile money services. We give the rank order lists of challenges faced by mobile money users in Figure 1. In Section 7 we compare the challenges identified through Twitter with the FII findings of the five countries that overlap from our study and the FII surveys.

\section{METHODOLOGY}

\subsection{Twitter Data Collection and Filtering}

We chose Bangladesh, Ghana, India, Kenya, Nigeria, Pakistan, Tanzania, South Africa, Senegal and Uganda as the initial countries

\begin{tabular}{|c|c|c|c|c|c|c|c|}
\hline Country & 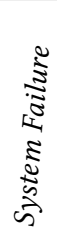 & 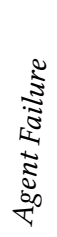 & 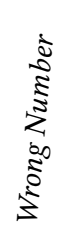 & 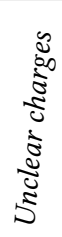 & 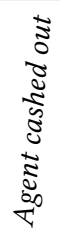 & 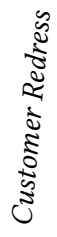 & 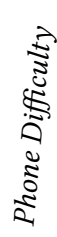 \\
\hline BD & 1 & 4 & 2 & 5 & 3 & - & - \\
\hline IN & 1 & 3 & - & 4 & - & 5 & 2 \\
\hline ID & 2 & 5 & 3 & 1 & - & - & 4 \\
\hline $\mathrm{KE}$ & 1 & 3 & 4 & - & 2 & 5 & - \\
\hline NG & 1 & 2 & 5 & - & - & 3 & 4 \\
\hline $\mathrm{PK}$ & 1 & 3 & 5 & 4 & 2 & - & - \\
\hline $\mathrm{TZ}$ & 1 & 3 & 5 & 2 & 4 & - & - \\
\hline UG & 1 & 3 & 4 & 2 & - & 5 & - \\
\hline
\end{tabular}

Figure 1: Top 5 Mobile Money Challenges from FII

of focus. A Python script ran continuously to collect data from September 2016 until June 2018 and collected 569, 301 tweets.

\begin{tabular}{|c|c|c|c|}
\hline Country & $\mathbf{N}$ & Popular handles & Tweets \\
\hline $\mathrm{BD}$ & 1 & Grameenphone & 349 \\
\hline $\mathrm{GH}$ & 9 & $\begin{array}{l}\text { MTNGhana, TigoGhana, } \\
\text { askvodafonegh }\end{array}$ & 39,462 \\
\hline IN & 13 & $\begin{array}{l}\text { Paytmcare, MobiKwikSWAT, } \\
\text { FreeCharge }\end{array}$ & 232,471 \\
\hline $\mathrm{KE}$ & 7 & $\begin{array}{l}\text { Safaricom_Care, TelkomKenya, } \\
\text { AIRTEL_KE }\end{array}$ & 107,429 \\
\hline NG & 7 & $\begin{array}{l}\text { mypaga, MyPocketMoni, } \\
\text { mypagacare }\end{array}$ & 19,379 \\
\hline PK & 16 & easypaisa, jazzpk, telenorpakistan & 21,234 \\
\hline $\mathrm{SN}$ & 2 & orange_sn, tigosn & 5,313 \\
\hline SA & 5 & rbjacobs, FNBSA, StandardBankZA & 84,625 \\
\hline $\mathrm{TZ}$ & 3 & $\begin{array}{l}\text { VodacomTanzania,Tigo_TZ, air- } \\
\text { tel_tanzania }\end{array}$ & 36,721 \\
\hline UG & 4 & mtnugcare, Airtel_Ug, africellUG & 22,318 \\
\hline
\end{tabular}

Figure 2: Summary of MFS Twitter handles and data parsed; $\mathbf{N}$ is the number of handles parsed for a country.

The typical Twitter customer care exchange begins with users sharing their problems by mentioning the Twitter handle of the MFS operator. A customer service representative responds and then a series of back and forth messages take place. We refer to such tweets where the MFS providers responds as actionable tweets. We decided to consider only English tweets which were actionable and hence decided to consider only Ghana, India, Kenya, Pakistan, South Africa and Uganda for our analysis.

\subsection{Qualitative Content Analysis}

From a total of 18,302 actionable tweets across the six countries, we randomly sampled 1500 tweets per country and labeled tweets which were MFS related, and then labeled them into higher and lower-level categories. In Figure 6, we show the counts of total MFS related tweets from the sample. For example, in the following 
tweet: ( "sent money to wrong number and I didn't know"), we labelled this tweet with the reversal and incorrect transaction as higher and lower-level categories respectively. We underwent several iterations to combine and condense similar labels, until no new modifications took place.

\begin{tabular}{|c|c|}
\hline $\begin{array}{l}\text { Higher Level } \\
\text { Category }\end{array}$ & Granular Category \\
\hline Account Access & phone locked, pin error, password error \\
\hline Fraud & SMS fraud, phone fraud, agent fraud, \\
\hline Reversal & $\begin{array}{l}\text { sent money to wrong person, incorrect } \\
\text { purchase of goods }\end{array}$ \\
\hline Service Error & SMS not received, balance not reflected \\
\hline Transaction Error & $\begin{array}{l}\text { deposit error, withdrawal error, cannot } \\
\text { complete transaction }\end{array}$ \\
\hline
\end{tabular}

Figure 3: Examples of higher and lower-level labels

\section{FINDINGS}

\subsection{Customer Support on Twitter}

Some operators even had a dedicated Twitter account for customer support queries (e.g., SafariCom used @safaricom_care and @safaricom). A typical Twitter discourse begins with the customer tweeting about an issue to the support Twitter handle. Some customers' tweets went unanswered; some tweets received just one response from support (either addressing the concern or redirecting the customer to a specific helpline); and some tweets lead to a back-and-forth conversation. When requesting more information from customers, support would ask them to share information via a Twitter direct message, but sometimes customers shared their personal information publicly on Twitter. When an issue is addressed, support would usually leave a tweet in the conversation confirming resolution of the issue or indicating that an action was taken, which also serves to promote the support as responsive and effective at handling customer issues.

\subsection{Issues Raised on Twitter}

Figure 4 shows the common issues we identified in our dataset and the number of tweets for each issue; we now discuss these issues.

5.2.1 Service and Account Access Issues. We saw several tweets about difficulties users face when using the smartphone app, SIM Toolkit App [9], or the USSD interface, include missing buttons ("what's up with the MySafcomApp; I can't use it coz I can't submit the service pin. No OK button?”), missing menu options ("@fazzCash this app main function is missing and fund transfer not possible in this $a p p$ "), and non-responsive interfaces ("@MobiKwikSWAT Pl check as to why one is unable to pay thru mobi wallet on hs18 app due to some error 404."). Users expected to receive the payment summary immediately and would tweet if the summary did not arrive as expected. In some cases users reported urgency, as the transactions were payments for services such as electricity and telephone, and the users were worried about the services getting disconnected because of delayed payment.

The challenges that users faced around account access were primarily due to users forgetting their mobile money PIN, trying

\begin{tabular}{|c|c|c|c|c|c|c|c|c|c|}
\hline 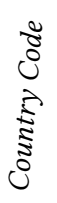 & 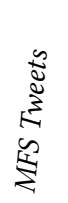 & 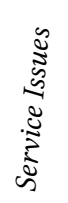 & 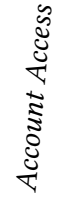 & 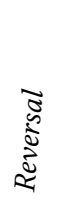 & 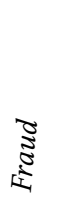 & 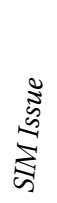 & 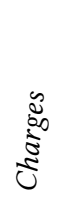 & 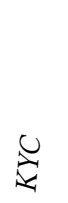 & 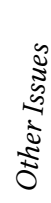 \\
\hline $\mathrm{GH}$ & 97 & 27 & 8 & 10 & 9 & 5 & 11 & 1 & 28 \\
\hline IN & 169 & 63 & 8 & 22 & 8 & 36 & 3 & 9 & 19 \\
\hline $\mathrm{KE}$ & 557 & 144 & 124 & 106 & 47 & 80 & 9 & 6 & 41 \\
\hline PK & 95 & 36 & 6 & 1 & 3 & 13 & 6 & 10 & 20 \\
\hline SA & 392 & 72 & 25 & 15 & 36 & 26 & 22 & 28 & 188 \\
\hline UG & 253 & 45 & 28 & 1 & 14 & 31 & 6 & 108 & 20 \\
\hline
\end{tabular}

Figure 4: Distribution of MFS related tweets in our manually coded dataset of 9,000 tweets (1,500 tweets per country)

multiple times, and locking their mobile money account after exceeding the allowed retries. To unlock their mobile money account, users need a Personal Unblocking Key (PUK number), which they enter in their phone. ("@AIRTEL_KE my simcard has blocked and I don't have puk code"). To get the PUK number, users tweet operators, who ask users for verification details via Twitter's direct message to provide the same.

5.2.2 Transactions Reversal. Users often sent money to an incorrect number ("@MTNGhana Please Is there a way one can use to retrieve money sent to a wrong number"), paid the wrong merchant or business ("I paid some sh1000 to the wrong business no.can u plz reverse."), paid for a wrong service ("I bought airtime instead of making a withdrawal"), went to a wrong agent (e.g., one agent tweeted "Kindly help the customer to reverse has withdraw from wrong agent") or entered a wrong number (e.g., "plz reverse. I sent to 2027805-01 instead of 2720805-01"). We found that transaction reversal requests were resolved quickly (e.g., within hours or days) when the transaction was between customers of a service provided by the same mobile money operator, but if the transaction involved a third party, resolving that issue took several days. To process reversal requests, most operators ask customers to verify their personal details and also ask the reason for reversal.

5.2.3 Fraud. When customers encounter fraud, they share details about the caller and the conversation. For example, one user reported that the caller "asked Airtel money \& Mpesa registrations. Also offering $50 \mathrm{~K}$ ". Most of the fraud reported by users is social engineering fraud, where fraudsters pose as customer care employee and engage customers in conversation and try to extract customers' personal identification information. Some users also reported fraudsters using threatening tone to divulge details. Unauthorized transaction was a common theme among fraud tweets in South Africa. Users reported their card being used for online transactions even though they did not lose their card or they do not remember sharing their card PIN with anyone. Most tweets about fraud were reports of fraud attempts and occurrences to providers, in which the user expected the provider to investigate and block the fraudster's number. 
5.2.4 Additional Issues. In addition to the three major themes, there were minor themes that emerged and those are discussed below:

Unexplained charges. Tweets regarding unexplained charges highlight the issue of lack of transparency in fees associated with mobile money services. Users wanted clarification on why they had been charged certain fees or why their balance was not what they expected, like the following user.

KYC. Customers have to provide identification documents to create a new mobile money account or continue using their account; failure to provide the documents results in account suspension or reduced transaction and balance limits on the account. Most of the tweets in our data about KYC issues were from Uganda. Many users had trouble successfully completing their registration or had to face account suspension because some checks in their documentation failed. Also, many users did not have national ID and they would tweet seeking assistance.

Query. Many customers, agents, and merchants used Twitter to ask customer care for information related to various issues including commission structure for an M-Pesa agent, specific helpline numbers, how to replace a SIM while retaining mobile money account, how to access account statement.

\subsection{Inter-Country Comparison}

Ghana, Kenya and Uganda. We found that the mobile money ecosystem in Kenya is well developed. Users can transact money, pay bills, get insured and even apply for loans. There were also $2 \%$ Latin Swahili tweets from our sample. In comparison, the mobile money ecosystem is growing rapidly in Ghana and it is relatively new in Uganda. SIM issues were quite common in these three countries since the ecosystem is MNO led. Emerging themes became apparent such as the mandatory law in Uganda to associate the National ID number with the SIM card, rendering all unverified simcards as invalid.

India. Demonetization in India led to an increase in digital wallet accounts [8]. There are a few MNO led initiatives for mobile money, but the market is dominated by mobile wallet services. In India, mobile wallet companies also serve as e-commerce providers. The majority of tweets referred to defective products or delayed deliveries, however, we do not consider these tweets as MFS related. 3\% of tweets in our sample were in Latin Hindi.

Pakistan. The MFS ecosystem in Pakistan is a hybrid of MNO led initiatives and digital wallet companies, but the majority of users use the former. Since mobile money is relatively new in Pakistan, users would ask questions and interact with customer care. $10 \%$ from our sample were in Latin and Persian Urdu.

South Africa. MNO led services were discontinued in 2016 and hence people use mobile banking applications in South Africa. [36] Most tweets were about customer service complaints, Users who were not satisfied when they had called or visited a physical branch would tweet their annoyance.

\section{DISCUSSION}

\subsection{Comparison with Other Studies}

Primarily we compare our findings with the MFS challenges outlined in Section 3. Overall, the main challenges that emerged from our analysis (service error, incorrect transaction, and fraud) match with the challenges identified by prior work, and our findings complement prior studies by identifying the nuances around these high-level challenges. The differences in the findings, we believe, are due to the differences in the underlying demographics of the users: Twitter users are primarily urban, whereas FII respondents could be either urban or rural. Other differences between previous findings and ours are mainly related to usability and agent issues.

\subsection{Twitter Analysis}

Twitter provides an opportunity to learn how users tweet and interact with customer care to get issues resolved. The process for addressing problems, such as verification, is through DM or a help line, but associated information, such as persistence of customers, is exposed. Other behaviors, such as tweeting on behalf of relatives or creating Twitter accounts just for reaching the customer support are also observed. But, there is bias in Twitter data as well. Twitter users are heavily urban and middle-class [35] and not representative of the population as a whole. It is probably the case that MFS challenges detected in Twitter data are relevant across the population. Another bias is that Twitter users are usually smartphone users. Also majority tweets were from male users in South Asia, with a slightly higher percentage of African female users. Also twitter usage and complaint resolution could vary across countries.

\subsection{Future Work}

Cross Cultural Studies. One limitation of this work is that we excluded countries where English was not the dominant twitter language. A natural extension of this work would be to consider those countries along with some countries in Francophone Africa, Latin America, and additional countries such as Indonesia.

Quantitative Studies. While this work allows us to identify important issues, our analysis does not give us a quantitative understanding of MFS challenges. We would like to develop an assessment of challenges that are robust across countries and MFS providers, and can also evaluate trends over time.

Improving MFS Technology. A question is whether this analysis can be utilized to help address the problems described. The numerous requests for transaction reversals suggest that there are challenges associated with the menu based user interfaces, and details in the requests show that there is a variety of different mistakes that can be made. Another area of research could be to mitigate fraud. One could measure the rates of fraud reports, develop a fraud classification system and develop early warnings of new scams as they spread.

\section{CONCLUSION}

We conducted an in-depth qualitative analysis of 9,000 mobile money complaint tweets from customers in six countries. Our analysis identified seven MFS issues and highlighted the details around these issues. Our findings also illuminated how MFS services are used differently in countries and how the nature of these challenges vary. We compared our results with prior studies that identified MFS challenges, and saw that our findings match and augment prior studies with greater nuance. Thus, we find Twitter as a rich data source that can provide insights about how people use mobile money and the challenges they face. 


\section{REFERENCES}

[1] Frank W. Agbola, Angelito Acupan, and Amir Mahmood. 2017. Does microfinance reduce poverty? New evidence from Northeastern Mindanao, the Philippines. fournal of Rural Studies 50 (Feb. 2017), 159-171. https://doi.org/10.1016/j.jrurstud. 2016.11.005

[2] Alexis Akwagyiram. 2012. Is social media changing how we complain? $B B C$ News (May 2012). https://www.bbc.com/news/uk-18081651

[3] Cynthia Andrews, Elodie Fichet, Yuwei Ding, Emma S. Spiro, and Kate Starbird 2016. Keeping Up with the Tweet-dashians: The Impact of 'Official' Accounts on Online Rumoring. In Proceedings of the 19th ACM Conference on ComputerSupported Cooperative Work \& Social Computing (CSCW'16). ACM, New York, NY, USA, 452-465. https://doi.org/10.1145/2818048.2819986

[4] Joshua E. Blumenstock, Michael Callen, Tarek Ghani, and Lucas Koepke. 2015. Promises and Pitfalls of Mobile Money in Afghanistan: Evidence from a Randomized Control Trial. In Proceedings of the Seventh International Conference on Information and Communication Technologies and Development (ICTD '15). ACM, New York, NY, USA, Article 15, 10 pages. https://doi.org/10.1145/2737856.2738031

[5] Sam Castle, Fahad Pervaiz, Galen Weld, Franziska Roesner, and Richard Anderson. 2016. Let's Talk Money: Evaluating the Security Challenges of Mobile Money in the Developing World. In Proceedings of the 7th Annual Symposium on Computing for Development (ACM DEV '16). ACM, New York, NY, USA, 4:1-4:10. https: //doi.org/10.1145/3001913.3001919

[6] Dipanjan Chakraborty, Mohd Sultan Ahmad, and Aaditeshwar Seth. 2017. Findings from a Civil Society Mediated and Technology Assisted Grievance Redressal Model in Rural India. In Proceedings of the Ninth International Conference on Information and Communication Technologies and Development (ICTD '17). ACM, New York, NY, USA, Article 2, 12 pages. https://doi.org/10.1145/3136560.3136574

[7] Dipanjan Chakraborty and Aaditeshwar Seth. 2015. Building Citizen Engagement into the Implementation of Welfare Schemes in Rural India. In Proceedings of the Seventh International Conference on Information and Communication Technologies and Development (ICTD '15). ACM, New York, NY, USA, Article 22, 10 pages. https://doi.org/10.1145/2737856.2738027

[8] Demonetization in India [n. d.]. Mobile wallets see a soaring growth postdemonetisation. http://bit.ly/2LDh0Zn

[9] Luigi Di Maggio and Corrado Guidobaldi. 2011. Method for accessing an additional service provided by an IC card toolkit applet. US Patent $8,036,706$

[10] Ficawoyi Donou-Adonsou and Kevin Sylwester. 2016. Financial development and poverty reduction in developing countries: New evidence from banks and microfinance institutions. Review of Development Finance 6, 1 (June 2016), 82-90. https://doi.org/10.1016/j.rdf.2016.06.002

[11] Paul Dunphy, Vasilis Vlachokyriakos, Anja Thieme, James Nicholson, John C. McCarthy, and Patrick Olivier. 2015. In Proceedings of the Symposium on Usable Privacy and Security (SOUPS). https://www.usenix.org/conference/soups2015/ proceedings/presentation/dunphy

[12] Sabine A. Einwiller and Sarah Steilen. 2015. Handling complaints on social network sites - An analysis of complaints and complaint responses on Facebook and Twitter pages of large US companies. Public Relations Review 41, 2 (June 2015), 195-204. https://doi.org/10.1016/j.pubrev.2014.11.012

[13] Elodie S Fichet, John J Robinson, Dharma Dailey, and Kate Starbird. 2016. Eyes on the Ground: Emerging Practices in Periscope Use during Crisis Events.. In ISCRAM. 1-10.

[14] FII [n. d.]. Reports, Financial Inclusion Insights by Intermedia. http://finclusion org/reports/

[15] Garren Gaut, Andrea Navarrete, Laila Wahedi, Paul van der Boor, Adolfo De Unánue, Jorge Díaz, Eduardo Clark, and Rayid Ghani. 2018. Improving Govern ment Response to Citizen Requests Online. In Proceedings of the 1st ACM SIGCAS Conference on Computing and Sustainable Societies (COMPASS '18). ACM, New York, NY, USA, Article 13, 10 pages. https://doi.org/10.1145/3209811.3209872

[16] Ishita Ghosh. 2012. The Mobile Phone As a Link to Formal Financial Services: Findings from Uganda. In Proceedings of the Fifth International Conference on Information and Communication Technologies and Development (ICTD '12). ACM, New York, NY, USA, 140-148. https://doi.org/10.1145/2160673.2160693

[17] Helix [n. d.]. Helix Institute of Digital Finance. http://helix-institute.com/

[18] Samia Ibtasam, Hamid Mehmood, Lubna Razaq, Jennifer Webster, Sarah Yu, and Richard Anderson. 2017. An Exploration of Smartphone Based Mobile Money Applications in Pakistan. In Proceedings of the Ninth International Conference on Information and Communication Technologies and Development (ICTD '17). ACM, New York, NY, USA, Article 1, 11 pages. https://doi.org/10.1145/3136560.3136571

[19] Samia Ibtasam, Lubna Razaq, Haider W. Anwar, Hamid Mehmood, Kushal Shah, Jennifer Webster, Neha Kumar, and Richard Anderson. 2018. Knowledge, Access, and Decision-Making: Women's Financial Inclusion In Pakistan. In Proceedings of the ACM SIGCAS Conference on Computing and Sustainable Societies (COMPASS) ACM, New York, NY, USA, Article 22, 12 pages. https://doi.org/10.1145/3209811. 3209819

[20] Doga Istanbulluoglu. 2017. Complaint handling on social media: The impact of multiple response times on consumer satisfaction. Computers in Human Behavior 74 (Sept. 2017), 72-82. https://doi.org/10.1016/j.chb.2017.04.016
[21] William Jack and Tavneet Suri. 2014. Risk Sharing and Transactions Costs : Evidence from Kenya's Mobile Money Revolution. American Economic Review (2014). https://doi.org/10.1257/aer.104.1.183

[22] Deepti Kumar, David Martin, and Jacki O’Neill. 2011. The Times They Are Achangin': Mobile Payments in India. In Proceedings of the SIGCHI Conference on Human Factors in Computing Systems (CHI '11). ACM, New York, NY, USA, 1413-1422. https://doi.org/10.1145/1978942.1979150

[23] Felix Kwizera, Isaac Markus, Purity Mugambi, and Abdigani Diriye. 2017. Use of Twitter Data Toward the Development of an English and Swahili Question Answering Agent for the Kenyan Customer Service Market. In Proceedings of the Ninth International Conference on Information and Communication Technologies and Development (ICTD '17). ACM, New York, NY, USA, Article 22, 5 pages. https://doi.org/10.1145/3136560.3136587

[24] Meghana Marathe, Jacki O'Neill, Paromita Pain, and William Thies. 2015. Revisiting CGNet Swara and Its Impact in Rural India. In Proceedings of the Seventh International Conference on Information and Communication Technologies and Development (ICTD '15). ACM, New York, NY, USA, Article 21, 10 pages. https://doi.org/10.1145/2737856.2738026

[25] Meghana Marathe, Jacki O'Neill, Paromita Pain, and William Thies. 2016. ICTEnabled Grievance Redressal in Central India: A Comparative Analysis. In Proceedings of the Eighth International Conference on Information and Communication Technologies and Development (ICTD '16). ACM, New York, NY, USA, Article 4, 11 pages. https://doi.org/10.1145/2909609.2909653

[26] Indrani Medhi, S.N. Nagasena Gautama, and Kentaro Toyama. 2009. A Comparison of Mobile Money-transfer UIs for Non-literate and Semi-literate Users. In Proceedings of the SIGCHI Conference on Human Factors in Computing Systems (CHI '09). ACM, New York, NY, USA, 1741-1750. https://doi.org/10.1145/1518701. 1518970

[27] Indrani Medhi, Aishwarya Ratan, and Kentaro Toyama. 2009. Mobile-Banking Adoption and Usage by Low-Literate, Low-Income Users in the Developing World. In Internationalization, Design and Global Development, Nuray Aykin (Ed.). Number 5623 in Lecture Notes in Computer Science. Springer Berlin Heidelberg, 485-494. http://link.springer.com/chapter/10.1007/978-3-642-02767-3_54 DOI: 10.1007/978-3-642-02767-3_54.

[28] Preeti Mudliar, Jonathan Donner, and William Thies. 2012. Emergent Practices Around CGNet Swara, Voice Forum for Citizen Journalism in Rural India. In Proceedings of the Fifth International Conference on Information and Communication Technologies and Development (ICTD '12). ACM, New York, NY, USA, 159-168. https://doi.org/10.1145/2160673.2160695

[29] Jacki O'Neill, Anupama Dhareshwar, and Srihari H. Muralidhar. 2017. Working Digital Money into a Cash Economy: The Collaborative Work of Loan Payment. Computer Supported Cooperative Work (CSCW) 26, 4 (01 Dec 2017), 733-768. https://doi.org/10.1007/s10606-017-9289-6

[30] Shereen Oraby, Pritam Gundecha, Jalal Mahmud, Mansurul Bhuiyan, and Rama Akkiraju. 2017. "How May I Help You?": Modeling Twitter Customer ServiceConversations Using Fine-Grained Dialogue Acts. In Proceedings of the 22Nd International Conference on Intelligent User Interfaces. ACM, 343-355. https: //doi.org/10.1145/3025171.3025191

[31] Joyojeet Pal, Priyank Chandra, Vaishnav Kameswaran, Aakanksha Parameshwar, Sneha Joshi, and Aditya Johri. 2018. Digital Payment and Its Discontents: Street Shops and the Indian Government's Push for Cashless Transactions. In Proceedings of the 2018 CHI Conference on Human Factors in Computing Systems (CHI '18). ACM, New York, NY, USA, Article 229, 13 pages. https://doi.org/10.1145/3173574.3173803

[32] Rowan Phipps, Shrirang Mare, Peter Ney, Jennifer Webster, and Kurtis Heimerl. 2018. ThinSIM-based Attacks on Mobile Money Systems. In Proceedings of the 1st ACM SIGCAS Conference on Computing and Sustainable Societies (COMPASS '18). ACM, New York, NY, USA, Article 23, 11 pages. https://doi.org/10.1145/3209811. 3209817

[33] Bradley Reaves, Nolen Scaife, Adam Bates, Patrick Traynor, and Kevin R. B. Butler. 2015. Mo(bile) Money, Mo(bile) Problems: Analysis of Branchless Banking Applications in the Developing World. In USENIX Security. 17-32.

[34] Johan Rewilak. 2017. The role of financial development in poverty reduction. Review of Development Finance 7, 2 (Dec. 2017), 169-176. https://doi.org/10.1016/ j.rdf.2017.10.001

[35] Taylor Shelton, Ate Poorthuis, and Matthew Zook. 2015. Social media and the city: Rethinking urban socio-spatial inequality using user-generated geographic information. Landscape and Urban Planning 142 (2015), 198-211.

[36] South Africa Mobile Money 2016. MTN is giving up on mobile money in South Africa. https://qz.com/783230

[37] Tavneet Suri and William Jack. 2016. The long term poverty and gender impacts of mobile money. Science 354, 6317 (2016), 1288-1292. https://doi.org/10.1126/ science.aah5309

[38] Women 2018. RFQ: Qualitative Research Firm in Indonesia. https://www. womensworldbanking.org/jobs/rfq-qualitative-research-firm-in-indonesia/

[39] World Bank 2015. Access to Financial Services - A Key to Lifting People Out of Poverty. World Bank. http://www.worldbank.org/en/news/press-release/2015/ 08/20/access-to-financial-services-key-to-lifting-people-out-of-poverty [Online; accessed 11. Jul. 2018]. 
[40] Sarah Yu and Samia Ibtasam. 2018. A Oualitative Exploration of Mobile Money in Ghana. In Proceedings of the 1st ACM SIGCAS Conference on Computing and Sustainable Societies (COMPASS '18). ACM, New York, NY, USA, Article 21, 10 pages. https://doi.org/10.1145/3209811.3209863
[41] Sarim Zafar, Usman Sarwar, Zafar Gilani, and Junaid Qadir. [n. d.]. In Proceedings of the ACM Symposium on Computing for Development (ACM DEV). 35:1-35:4. https://doi.org/10.1145/3001913.3006644 